\title{
Diagnosis of broad complex tachycardias with ambulatory monitoring of atrial electrography
}

\author{
D P LIPKIN, E J PERRINS, L M SHAPIRO, LESLEY LUDGATE, K M FOX
}

\begin{abstract}
Simultaneous 24 hour ambulatory monitoring of the surface electrocardiogram and the intra-atrial electrocardiogram was evaluated in eight patients with broad complex tachycardia. The technique using a J shaped atrial pacing wire permitted a distinction between ventricular tachycardia and supraventricular tachycardia in all eight patients. Formal electrophysiological testing would still be required, however, in patients in whom diagnostic doubt remained.
\end{abstract}

\section{Introduction}

The QRS complex in supraventricular tachycardia is usually narrow. Occasionally, conduction to the ventricle during supraventricular tachycardia may occur with bundle branch block configuration. In these circumstances the QRS complex will be broad $(\geqslant 0.12 \mathrm{~s})$, and supraventricular tachycardia may then be difficult to distinguish from ventricular tachycardia. The presence of atrioventricular dissociation during the arrhythmia strongly suggests a ventricular origin of the tachycardia. Although atrioventricular dissociation during broad complex tachycardia may be shown with 12 lead electrocardiography, recordings obtained during Holter monitoring often do not permit easy recognition of the $P$ wave. As the distinction between ventricular and supraventricular arrhythmias is clinically important we evaluated the use of ambulatory monitoring of the surface electrocardiogram and intra-atrial electrogram simultaneously.

National Heart Hospital, London W1M 8BA

D P LIPKIN, BSC, MRCP, medical registrar E J PERRINS, BSC, MRCP, senior registrar L M SHAPIRO, MD, MRCP, senior registrar LESLEY LUDGATE, pacing technician

K M FOX, MD, MRCP, consultant cardiologist

Correspondence and requests for reprints to: Dr D P Lipkin.

\section{Methods and results}

We studied eight patients with previously recorded broad complex tachycardia. They had all given their written consent. A disposable temporary bipolar J shaped atrial pacing wire (Cordis 410) was positioned by fluoroscopy in the right atrial appendage. This was done via a right subclavian vein puncture by withdrawing and anteriorly rotating the wire placed initially in the right atrium. The two leads of the pacing catheter were connected with a standard pacing connector to one channel of a standard unmodified two channel ambulatory recorder (Reynolds Tracker), the other channel being connected to lead CM5. The tapes were analysed using the Reynolds Pathfinder system. No restrictions were placed on any patient's activity during the 24 hour period of recording.

The apparatus was comfortable to wear and did not interfere with general activity or sleeping. Satisfactory atrial electrograms were recorded in all patients. No leads were displaced, and no other complications occurred.

In six patients ventricular tachycardia was confirmed (figure) by the presence of $\mathbf{A}$ waves (representing atrial activity) which were dissociated from the broad ventricular complexes seen on the recording from the surface lead. In the two other patients both ventricular tachycardia and supraventricular tachycardia conducted with aberrancy were recognised.

\section{Discussion}

Ambulatory monitoring of the atrial electrocardiogram appears to be a simple, inexpensive procedure. It provides an

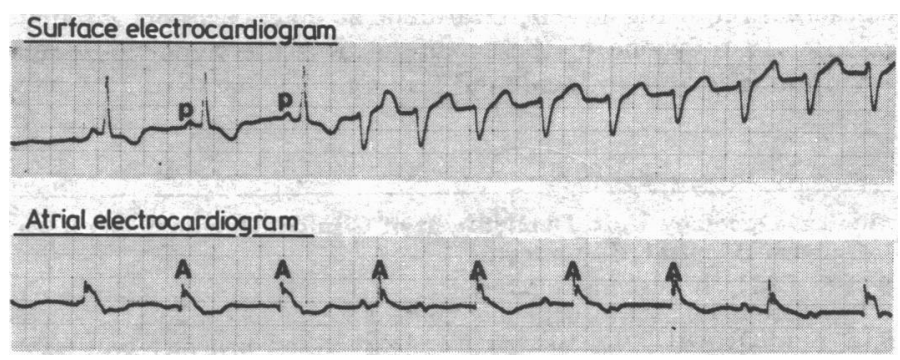

Top: Surface electrocardiogram (lead CM5) showing three sinus beats followed by an episode of broad complex tachycardia without recognisable $\mathbf{P}$ waves or fusion beats. Bottom: intra-atrial electrogram showing atrial activity (A waves) dissociated from the broad complex tachycardia. 
easy method of identifying atrial activity and therefore facilitates the diagnosis of complex tachycardia. Reliable recording of atrial activity had previously been obtained with intraoesophageal electrodes. ${ }^{1}$ Prolonged monitoring by this method, however, is uncomfortable for the patient and may be unsatisfactory owing to movement of the electrodes during oesophageal peristaltic activity. This technique has not been applied to ambulatory monitoring.

Ambulatory monitoring of the atrial electrogram has recently been reported by Davies et al, who used a specially manufactured temporary screw in atrial electrode. ${ }^{2}$ Fixation of the electrode tip, however, is relatively complicated and this technique is therefore not suitable for those who are not trained in atrial pacing. In contrast, the bipolar J shaped atrial pacing wire that we used is available in most district general hospitals and specialised skill is not required to insert the lead and interpret the traces.

This technique would also be of use in elucidating arrhythmias in patients with pre-existing conduction disease-for example, bundle branch block-and in investigating patients with suspected bradycardia. In one patient a pause of $1.9 \mathrm{~s}$ in the surface electrocardiogram was found to be due to two closely coupled blocked atrial ectopic beats.

We must emphasise that the presence of one to one atrioventricular activation during an episode of tachycardia does not rule out ventricular tachycardia as retrograde atrioventricular conduction may be occurring. Furthermore, atrioventricular dissociation may occur in the absence of ventricular tachycardia with nodal tachycardia and retrograde atrioventricular block. Formal electrophysiological testing will still be required in patients in whom diagnostic doubt remains.

\section{References}

1 Jenkins JM, Delob W, Arzbaecher RC. Computer diagnosis of supraventricular and ventricular arrhythmias. Circulation 1979;60:977-85.

2 Davies DW, Crick JCP, Holt PM, Curry PVL, Sowton E. Benefits of long term recording of the atrial electrogram. PACE 1983;6:387.

(Accepted 22 March 1984)

\title{
Paraneoplastic hypercalcaemia in ovarian carcinoma
}

\author{
S G ALLAN， S P LOCKHART， R C F LEONARD， J F SMYTH
}

\begin{abstract}
Five patients were seen in whom a raised serum calcium concentration was associated with ovarian carcinoma (clear cell in two cases, cystadenocarcinoma in three). None showed evidence of metastases in bone. The hypercalcaemia occurred as a paraneoplastic phenomenon, but biochemical studies suggested the production of a parathyroid-hormone-like substance. One patient remained free of symptoms of her hypercalcaemia throughout.

Paraneoplastic hypercalcaemia due to ovarian carcinoma may be more common than generally recognised and present as a life threatening condition requiring urgent treatment.
\end{abstract}

\section{Introduction}

Hypercalcaemia may occur in patients with malignant diseases, usually resulting from multiple bone metastases. The multiple bone metastases are rare in patients with ovarian carcinoma. ${ }^{12}$ We describe five patients with ovarian carcinoma presenting with hypercalcaemia as a paraneoplastic phenomenon, not associated with bone metastases. Two patients had hypercalcaemia requiring urgent treatment at diagnosis and three at relapse. In only one of these patients was the hypercalcaemia asymptomatic and an incidental finding.

\footnotetext{
Medical Oncology Unit, Department of Clinical Oncology, Western General Hospital, Edinburgh

S G ALLAN, MB, MRCP, lecturer

S P LOCKHART, MA, MRCP, senior house officer

R C F LEONARD, MD, MRCP, senior lecturer and honorary consultant J F SMYTH, MD, FRCP, professor and honorary consultant

Correspondence to: Professor J F Smyth, University Department of Clinical Oncology, Western General Hospital, Edinburgh EH4 2XU.
}

\section{Case reports}

Case 1-A 40 year old woman presented with a four week history of nausea, vomiting, anorexia, and polyuria. On examination she was found to be dehydrated and to have a large mass in the lower abdomen and two supraclavicular lymph nodes. The serum calcium concentration was $3.96 \mathrm{mmol} / 1(15.8 \mathrm{mg} / 100 \mathrm{ml})$ and she was given fluids, frusemide, and mithramycin before laparotomy. A widespread stage IV ovarian carcinoma was largely resected from the abdomen, and histological examination showed a papillary cystadenocarcinoma. Her serum calcium concentration then fell to normal and she was given chemotherapy. Four months later her disease progressed and the serum calcium concentration rose to $2.68 \mathrm{mmol} / 1(10.7 \mathrm{mg} / 100 \mathrm{ml})$ (table).

Case 2-This patient aged 35, had presented in 1979 with a stage IA clear cell carcinoma of ovary. This was resected, and she remained well until July 1983, when she gave a four month history of worsening nausea, vomiting, anorexia, and lethargy. On examination she was found to be drowsy, dehydrated, and cachectic with gross ascites. Serum calcium concentration was $3.14 \mathrm{mmol} / 1(12.6 \mathrm{mg} / 100 \mathrm{ml})$ (table). Recurrent ovarian carcinoma was detected in pelvis and liver. The hypercalcaemia responded to prednisolone.

Case 3-A 69 year old woman had undergone laparotomy for a stage IIA serous cystadenocarcinoma of ovary in 1979 followed by abdominal radiotherapy. She had remained well until January 1983, when she gave a one month history of persistent vomiting, thirst, and polyuria. Subacute intestinal obstruction was diagnosed initially, but a raised serum calcium concentration was then discovered $(3.49 \mathrm{mmol} / 1 ; 14.0 \mathrm{mg} / 100 \mathrm{ml})$. She was found to have recurrent carcinoma in pelvis and lungs. The hypercalcaemia and symptoms responded to prednisolone.

Case 4-A 64 year old woman had had a stage IIB clear cell carcinoma excised in 1981. In March 1983 she developed venous thrombosis but also complained of anorexia, nausea, constipation, thirst, and nocturia (thrice nightly). Recurrent carcinoma was found in pelvis and lungs and hypercalcaemia $(3.06 \mathrm{mmol} / 1 ; 12.2 \mathrm{mg} / 100 \mathrm{ml})$ confirmed. The hypercalcaemia and symptoms responded to prednisolone.

Case 5-This patient, aged 54, had undergone partial excision of a stage III papillary cystadenocarcinoma in 1982. Her serum calcium concentration had coincidentally been noted to be high $(2.72 \mathrm{mmol} / 1$; $10.9 \mathrm{mg} / 100 \mathrm{ml}$ ) when she presented for chemotherapy. The con- 